\title{
C. BelabBACI
}

\section{SPECTRAL RADIUS OF S-ESSENTIAL SPECTRA}

\section{Belabbaci. Spectral radius of S-essential spectra, Mat. Stud. 54 (2020), 91-97.}

In this paper, we study the spectral radius of some S-essential spectra of a bounded linear operator defined on a Banach space. More precisely, via the concept of measure of noncompactness, we show that for any two bounded linear operators $T$ and $S$ with $S$ non zero and non compact operator the spectral radius of the S-Gustafson, S-Weidmann, S-Kato and S-Wolf essential spectra are given by the following inequalities

$$
\frac{\beta(T)}{\alpha(S)} \leq r_{e, S}(T) \leq \frac{\alpha(T)}{\beta(S)},
$$

where $\alpha($.$) stands for the Kuratowski measure of noncompactness and \beta($.$) is defined in [11].$ In the particular case when the index of the operator $S$ is equal to zero, we prove the last inequalities for the spectral radius of the S-Schechter essential spectrum. Also, we prove that the spectral radius of the S-Jeribi essential spectrum satisfies inequalities (2) when the Banach space $X$ has no reflexive infinite dimensional subspace and the index of the operator $S$ is equal to zero (the S-Jeribi essential spectrum, introduced in [7] as a generalisation of the Jeribi essential spectrum).

1. Introduction. The spectral theory of operators pencils $(\lambda S-T)$ (operator-valued functions of a complex argument) play a crucial role in many branches of mathematical physics see, for example, $[2,5,4,12]$.

The purpose of this paper is to study the spectral radius of some S-essential spectra of a bounded linear operator on a Banach space $X$. More precisely, we give a localization of the spectral radius of the $S$-essential spectra of an operator $T$ via the concept of measure of noncompactness when $S$ is a non compact and non zero bounded linear operator and $T$ is any bounded linear operator. This work continues research begun in [7], here we aim to prove that the spectral radius of the S-Gustafson, S-Weidmann, S-Kato and S-Wolf essential spectrum is given by the following inequalities:

$$
\frac{\beta(T)}{\alpha(S)} \leq r_{e, S}(T) \leq \frac{\alpha(T)}{\beta(S)},
$$

where $\alpha($.$) stands for the Kuratowski measure of noncompactness and \beta($.$) is defined in the$ next section.

When the index of the operator $S$ is equal to zero, then the inequalities (2) holds for the S-Schechter essential spectrum. Also, we prove the above inequalities (2) for the S-Jeribi essential spectrum, introduced in [7] as a generalisation of the Jeribi essential spectrum, in the particular case when the Banach space $X$ has no reflexive infinite dimensional subspace.

2010 Mathematics Subject Classification: 46B50, 47A13, 47A53, $47 \mathrm{H} 08$.

Keywords: S-essential spectra; measure of noncompactness; spectral radius; Fredholm operators. doi:10.30970/ms.54.1.91-97

(C) C. Belabbaci, 2020 
The paper is organised as follows. Section 2 contains an overview of the necessary background and Section 3 contains the main results.

2. Preliminaries. The notion of measure of non-compactness have been successfully applied in many different domains in mathematics; in fixed point theory, differential equations, functional equations, integral and integro-differential equations, etc. (see for example $[14,13,3,8]$ ). In order to recall this notion denote by $X$ a Banach space, $M_{X}$ the set of all nonempty and bounded subsets of $X$. The Kuratowski measure of noncompactness of a set $A$ in $M_{X}$, denoted $\alpha(A)$, is defined by

$$
\alpha(A)=\inf \left\{\varepsilon>0: \quad A \subset \bigcup_{i=1}^{n} S_{i}, \quad S_{i} \subset X, \quad \operatorname{diam} S_{i} \leq \varepsilon, \quad i=1, \ldots, n\right\} .
$$

Denote by $\mathcal{L}(X)$ the space of all bounded linear operators of $X$ into $X$ and let $T \in \mathcal{L}(X)$. The Kuratowski measure of noncompactness of an operator $T$ is defined as follows

$$
\alpha(T)=\inf \left\{k \geq 0: \alpha(T(A)) \leq k \alpha(A) \text { for any set } A \in M_{X}\right\},
$$

it can be equivalently defined as

$$
\alpha(T)=\sup \left\{\frac{\alpha(T(A))}{\alpha(A)} ; A \in M_{X}, \alpha(A)>0\right\} .
$$

We define the following nonnegative quantity, $\beta(T)$, as (see [11])

$$
\beta(T)=\inf \left\{\frac{\alpha(T(A))}{\alpha(A)} ; A \in M_{X}, \alpha(A)>0\right\} .
$$

In what follows, we give some fundamental properties of $\alpha, \beta$ already given in [11].

Proposition 1. Let $T, S$ be in $\mathcal{L}(X)$. Then the following claims hold:

1. $\alpha(\lambda T)=|\lambda| \alpha(T)$ and $\beta(\lambda T)=|\lambda| \beta(T)$ for all $\lambda \in \mathbb{C}$.

2. $|\alpha(T)-\alpha(S)| \leqslant \alpha(T+S) \leqslant \alpha(T)+\alpha(S)$.

3. $\beta(T)-\alpha(S) \leqslant \beta(T+S) \leqslant \beta(T)+\alpha(S)$.

4. $\alpha(T \circ S) \leqslant \alpha(T) \alpha(S)$ and $\beta(T \circ S) \geqslant \beta(T) \beta(S)$.

5. $\alpha(T)=0$ if and only if $T$ is compact.

Recall now the following important operators. Let $T \in \mathcal{L}(X)$, denote by $R(T)$ the range of $T$ and $\operatorname{ker}(T)$ the null space of $T$. The nullity of $T, n(T)$, is defined as the dimension of $\operatorname{ker}(T)$ and the deficiency of $T, d(T)$, is defined as the codimension of the range $R(T)$ in $X$. An operator $T \in \mathcal{L}(X)$ is called upper semi Fredholm operator if $R(T)$ is closed and $n(T)$ is finite, it is called lower semi Fredholm operator if $d(T)$ is finite. The set of all upper (respectively lower) semi Fredholm operators is denoted respectively by $\Phi_{+}(X)$ and $\Phi_{-}(X)$. $\Phi(X)=\Phi_{+}(X) \cap \Phi_{-}(X)$ is called the set of Fredholm operators and $\Phi_{ \pm}(X)=\Phi_{+}(X) \cup \Phi_{-}(X)$ is called the set of semi Fredholm operators. The index of an operator $T \in \Phi_{ \pm}(X)$ is defined as ind $(T)=n(T)-d(T)$. The following properties are well known.

Proposition 2. Let $T \in \mathcal{L}(X)$ and $T^{*}$ be the adjoint operator of $T$. Then the following claims hold: 
1. $\beta(T)>0$ if and only if $T \in \Phi_{+}(X)$;

2. $\beta\left(T^{*}\right)>0$ if and only if $T \in \Phi_{-}(X)$.

Let $T, S \in \mathcal{L}(X)$ with $S$ non zero. We define the S-resolvent set of $T$ by

$$
\rho_{S}(T)=\{\lambda: \lambda S-T \text { has a bounded inverse }\}
$$

and the S-spectrum of $T$ by $\sigma_{S}(T)=\mathbb{C} \backslash \rho_{S}(T)$. There are several definitions of the S-essential spectrum of a bounded linear operator on a Banach space, in this paper we are interested with the following definitions:

$\begin{array}{ll}\text { S-Wolf } & \sigma_{e_{1, S}}(T)=\{\lambda \in \mathbb{C}:(\lambda S-T) \notin \Phi(X)\} \\ \text { S-Gustafson } & \sigma_{e_{2, S}}(T)=\left\{\lambda \in \mathbb{C}:(\lambda S-T) \notin \Phi_{+}(X)\right\} ; \\ \text { S-Weidmann } & \sigma_{e_{3, S}}(T)=\left\{\lambda \in \mathbb{C}:(\lambda S-T) \notin \Phi_{-}(X)\right\} ; \\ \text { S-Kato } & \sigma_{e_{4, S}}(T)=\left\{\lambda \in \mathbb{C}:(\lambda S-T) \notin \Phi_{ \pm}(X)\right\} ; \\ \text { S-Schechter } & \sigma_{e_{5, S}}(T)=\mathbb{C} / \Phi_{0}(T) ; \\ & \Phi_{0}(T)=\{\lambda:(\lambda S-T) \in \Phi(X) \text { with ind }(\lambda S-T)=0\} \\ \text { S-Jeribi } & \sigma_{e_{j, S}}(T)=\bigcap_{W \in \mathcal{W}_{*}(X)} \sigma_{S}(T+W) ;\end{array}$

where $\mathcal{W}_{*}(X)$ stands for each one of the sets of weakly compact operators $\mathcal{W}(X)$ and strictly singular operators $\mathcal{S}(X)$. The following inclusion $\sigma_{e_{j, S}}(T) \subset \sigma_{e_{5, S}}(T)$ is always satisfies since the set $\mathcal{W}_{*}(X)$ contains the set of compact operators $\mathcal{K}(X)$. In general, we have the following inclusions:

$$
\sigma_{e_{4, S}}(T)=\sigma_{e_{2, S}}(T) \cap \sigma_{e_{3, S}}(T) \subseteq \sigma_{e_{1, S}}(T) \subseteq \sigma_{e_{5, S}}(T) .
$$

Note that if $S$ is the identity operator, we recover the usual definition of essential spectra of a bounded linear operator $T ; \sigma_{e_{i}}(T)$ for $i=1, \ldots, 5$ and $\sigma_{j}(T)$. The interested reader may find further results on the essential spectra and S-essential spectra in [10], [9]. When the S-essential spectrum is a non empty set, we define the $\mathrm{S}$-essential spectral radius of $T$ as follows

$$
r_{e_{i}, S}(T)=\sup \left\{|\lambda|: \lambda \in \sigma_{e_{i}, S}(T)\right\} ; \quad i=1, \ldots, 5, j .
$$

3. Main results. In this section we present our main results. We give a localization for the spectral radius of some S-essential spectra. In the particular, the S-Gustafson, S-Wolf, S-Weidmann, S-Kato, S-Schechter and S-Jeribi essential spectrum.

We begin by the spectral radius of the S-Gustafson essential spectrum. We have the following main theorem.

Theorem 1. Let $T, S$ be two bounded operators on $X$, with $S$ nonzero and noncompact. Then the radius of the $S$-Gustafson essential spectrum is given by

$$
\frac{\beta(T)}{\alpha(S)} \leq r_{e_{2}, S}(T) \leq \frac{\alpha(T)}{\beta(S)}
$$

Proof. Let us consider $\lambda \in \mathbb{C}$ such that $|\lambda|>\frac{\alpha(T)}{\beta(S)}$. Then we have $\beta(\lambda S)-\alpha(T)>0$. Using respectively assertion (1) of Proposition 1 and assertion (2) of Proposition 2, we obtain $(\lambda S-T) \in \Phi_{+}(X)$, i.e. $\lambda$ is not in the S-Gustafson essential spectrum $\sigma_{e_{2}, S}(T)$. That is, if $\lambda$ belongs to $\sigma_{e_{2}, S}(T)$ then $|\lambda| \leq \frac{\alpha(T)}{\beta(S)}$. Hence

$$
r_{e_{2}, S}(T) \leq \frac{\alpha(T)}{\beta(S)}
$$


To prove the last inequality, let $\lambda \in \mathbb{C}$ such that $|\lambda|<\frac{\beta(T)}{\alpha(S)}$. Then we have $\beta(T)-\alpha(\lambda S)>0$. Using assertion (1) of Proposition 1 and assertion (2) of Proposition 2 respectively, we get $(\lambda S-T) \in \Phi_{+}(X)$, i.e. $\lambda \notin \sigma_{e_{2}, S}(T)$. Thus

$$
\lambda \in \sigma_{e_{2}, S}(T) \Rightarrow|\lambda| \geq \frac{\beta(T)}{\alpha(S)} .
$$

So, we have

$$
r_{e_{2}, S}(T) \geq \frac{\beta(T)}{\alpha(S)}
$$

From the above inequalities (4) and (5), we deduce that

$$
\frac{\beta(T)}{\alpha(S)} \leq r_{e_{2}, S}(T) \leq \frac{\alpha(T)}{\beta(S)}
$$

We shall prove Theorem 1 for the S-Weidmann essential spectrum, but first we need to prove the following lemma:

Lemma 1. Let $T, S$ be two bounded operators on $X$, with $S$ nonzero and noncompact. Then we have

$$
\frac{\alpha(T)}{\beta(S)}=\frac{\alpha\left(T^{*}\right)}{\beta\left(S^{*}\right)}
$$

where $T^{*}, S^{*}$ are the adjoint operator of $T$ and $S$, respectively.

Proof. The following inequalities are well known (see [10])

$$
\frac{1}{2} \alpha(T) \leq \alpha\left(T^{*}\right) \leq \alpha(T) .
$$

For any bounded set $A$ in $X$ with $A$ non relatively compact set, we have

$$
\frac{1}{2} \frac{\alpha(S(A))}{\alpha(A)} \leq \frac{\alpha\left(S^{*}(A)\right)}{\alpha(A)} \leq \frac{\alpha(S(A))}{\alpha(A)} .
$$

This implies that

$$
\inf \left\{\frac{\alpha\left(S^{*}(A)\right)}{\alpha(A)}, \alpha(A)>0\right\} \leq \frac{1}{2} \inf \left\{\frac{\alpha(S(A))}{\alpha(A)}, \alpha(A)>0\right\}
$$

and

$$
\inf \left\{\frac{\alpha(S(A))}{\alpha(A)}, \alpha(A)>0\right\} \leq \inf \left\{\frac{\alpha\left(S^{*}(A)\right)}{\alpha(A)}, \alpha(A)>0\right\} .
$$

By definition of $\beta$, we get $\beta\left(S^{*}\right) \leq \frac{1}{2} \beta(S)$ and $\beta(S) \leq \beta\left(S^{*}\right)$ i.e

$$
\frac{2}{\beta(S)} \leq \frac{1}{\beta\left(S^{*}\right)} \leq \frac{1}{\beta(S)}
$$

Timing the inequalities (7) and (6) we get the results.

The following main theorem holds. 
Theorem 2. Let T, $S$ be two bounded operators on $X$, with $S$ non zero and non compact. Then the spectral radius of the $S$-essential spectra is given by

$$
\frac{\beta(T)}{\alpha(S)} \leq r_{e_{i}, S}(T) \leq \frac{\alpha(T)}{\beta(S)} \quad \text { for } i=3,4,1
$$

Proof. First, we prove the inequalities (8) for the S-Weidmann essential spectrum. Let $\lambda \in \mathbb{C}$ such that $|\lambda|<\frac{\beta\left(T^{*}\right)}{\alpha\left(S^{*}\right)}$, then $\beta\left(T^{*}\right)-\alpha\left(\lambda S^{*}\right)>0$. From proposition (2), this implies that $\beta\left((\lambda S-T)^{*}\right)>0$. Using properties of $\beta$, we see that $(\lambda S-T) \in \Phi_{-}(X)$. i.e $\lambda \notin \sigma_{e_{3}, S}(T)$. Therefore, if $\lambda \in \sigma_{e_{3}, S}(T)$ then $|\lambda| \geq \frac{\alpha\left(T^{*}\right)}{\beta\left(S^{*}\right)}$. By the use of Lemma (1) we conclude that

$$
r_{e_{3}, S}(T) \geqslant \frac{\alpha(T)}{\beta(S)} \text {. }
$$

In order to prove the other inequality, let us take $\lambda \in \mathbb{C}$ such that $|\lambda|>\frac{\alpha\left(T^{*}\right)}{\beta\left(S^{*}\right)}$. Then $\beta\left(\lambda S^{*}\right)>\alpha\left(T^{*}\right)$. From assertion (1) of proposition (1) and assertion (2) of proposition (2) respectively, we get $(\lambda S-T) \in \Phi_{-}(X)$. This means that $\lambda \notin \sigma_{e_{3}, S}(T)$. Consequently, by the use of Lemme (1) we get the following implication

$$
\lambda \in \sigma_{e_{3}, S}(T) \Rightarrow|\lambda| \leqslant \frac{\alpha(T)}{\beta(S)}
$$

Hence

$$
r_{e_{3}, S}(T) \leqslant \frac{\alpha(T)}{\beta(S)} .
$$

From the two inequalities (9) and (11), we get a localization for the S-Weidmann essential spectrum

$$
\frac{\beta(T)}{\alpha(S)} \leq r_{e_{3}, S}(T) \leq \frac{\alpha(T)}{\beta(S)}
$$

Now, we prove the above inequalities for the S-Wolf essential spectrum. From the inclusion $\sigma_{e_{2}, S}(T) \subset \sigma_{e_{1}, S}(T)$ we have $r_{e_{2}, S}(T) \leq r_{e_{1}, S}(T)$. So the following inequality holds

$$
\frac{\beta(T)}{\alpha(S)} \leq r_{e_{1}, S}(T)
$$

For the last inequality, let us suppose $\lambda \in \mathbb{C}$ such that $|\lambda|>\frac{\alpha(T)}{\beta(S)}$. Then using Lemme (1), we have $|\lambda|>\frac{\alpha(T)}{\beta(S)}=\frac{\alpha\left(T^{*}\right)}{\beta\left(S^{*}\right)}$. i.e $|\lambda| \beta(S)>\alpha(T)$ and $|\lambda| \beta\left(S^{*}\right)>\alpha\left(T^{*}\right)$. Using properties of $\beta$ we get $\beta(\lambda S-T)>0$ and $\beta\left(\lambda S^{*}-T^{*}\right)>0$. This implies that $(\lambda S-T) \in \Phi_{+}(X) \cap \Phi_{-}(X)=$ $\Phi(X)$. Hence $\lambda \notin \sigma_{e_{1}, S}(T)$. Therefore

$$
\lambda \in \sigma_{e_{1}, S}(T) \Rightarrow|\lambda| \leq \frac{\alpha(T)}{\beta(S)}
$$

Consequently we have the following inequality

$$
r_{e_{1}, S}(T) \leq \frac{\alpha(T)}{\beta(S)}
$$

From the two inequalities (13) and (12), we get the results. 
In what follows, we prove the above localization for the S-Kato essential spectrum. From the equality $\sigma_{e_{4}, S}(T)=\sigma_{e_{2}, S}(T) \cap \sigma_{e_{3}, S}(T)$, we infer that $r_{e_{4}, S}(T) \leqslant \frac{\alpha(T)}{\beta(S)}$. Now it suffices to show that $r_{e_{4}, S}(T) \geq \frac{\beta(T)}{\alpha(S)}$. Suppose that $|\lambda|<\frac{\beta(T)}{\alpha(S)}$, then using properties of $\beta$ we see that $(\lambda S-T) \in \Phi_{+}(X)$. Consequently $\lambda \notin \sigma_{e_{4}, S}(T)$. We deduce that, if $\lambda$ belongs to $\sigma_{e_{4}, S}(T)$ then $|\lambda| \geq \frac{\alpha(T)}{\beta(S)}$. Hence $r_{e_{4}, S}(T) \geq \frac{\alpha(T)}{\beta(S)}$.

In the following theorem, we prove formulae (2) for the S-Schechter essential spectrum.

Theorem 3. Let T, $S$ be two bounded operators on a Banach space $X$, with $S$ non zero and non compact. Suppose that ind $S=0$. Then the spectral radius of the $S$-Schechter essential spectrum is given by

$$
\frac{\beta(T)}{\alpha(S)} \leq r_{e_{5}, S}(T) \leq \frac{\alpha(T)}{\beta(S)}
$$

Proof. It follows immediately from the inclusion $\sigma_{e_{1, S}}(T) \subset \sigma_{e_{5, S}}(T)$ that $\frac{\beta(T)}{\alpha(S)} \leqslant r_{e_{5}, S}(T)$. In order to prove the last inequality, let us take $\lambda \in \mathbb{C}$ such that $|\lambda| \geqslant \frac{\alpha(T)}{\beta(S)}$. Using $[1$, Theorem 2.2], we obtain that $(\lambda S-T) \in \Phi_{+}(X)$ and $\operatorname{ind}(\lambda S-T)=\operatorname{ind}(\lambda S)$. According to the hypothesis ensuring ind $S=0$, it follows that $(\lambda S-T) \in \Phi(X)$ with ind $(\lambda S-T)=0$. Hence $\lambda \in \sigma_{e_{1, S}}(T)$ implies that $|\lambda| \leq \frac{\alpha(T)}{\beta(S)}$. We deduce that $r_{e_{5}, S}(T) \leq \frac{\alpha(T)}{\beta(S)}$.

We end this section by the spectral radius of the S-Jeribi essential spectrum. In the definition of the S-Jeribi essential spectrum, we restrict $K$ belonging to $\mathcal{W}(X)$ only since $X$ is a Banach space. The other main results is the following theorems:

Theorem 4. Let $X$ be a Banach space which has no reflexive infinite dimensional subspaces and $T \in \mathcal{L}(X)$. Then we have

$$
\sigma_{e_{i, S}}(T) \subset \sigma_{j, S}(T), \quad i=1, \ldots, 4
$$

Proof. Reasoning in the same way as the proof of [6, Theorem 3.3].

The following main theorem gives a localization for the spectral radius of the S-Jeribi essential spectrum in the particular case when the Banach space $X$ has no reflexive infinite dimensional subspaces.

Theorem 5. Let $X$ be a Banach space which has no reflexive infinite dimensional subspaces, $T, S \in \mathcal{L}(X)$ and ind $S=0$. Then the spectral radius of the $S$-Jeribi essential spectrum satisfies formulae (2).

Proof. From the inclusion $\sigma_{e_{j, S}}(T) \subset \sigma_{e_{5, S}}(T)$, we infer that $r_{e_{j, S}}(T) \leq \frac{\alpha(T)}{\beta(S)}$. The last inequality is satisfies by using Theorem 4 . 
1. B. Abdelmoumen, H. Baklouti, Perturbation results on semi-Fredholm operators and applications, J. Inequal. Appl., 2009, Article ID 284526, 1-13.

2. F. Abdmouleh, A. Ammar, A. Jeribi, Stability of the S-essential spectra on a Banach space, Math. Slovaca, 63 (2013), 299-320.

3. R. Akhmerov, M. Kamenski, A. Potapov, A. Rodkina, B. Sadovskii, Measures of noncompactness and condensing operators, Operator Theory: Advances and Applications, 55, Birkhäuser Verlag, Basel, Boston, Berlin, 1992. (in Russian)

4. A. Ammar, B. Boukettaya, A. Jeribi, Stability of the S-left and S-right essential spectra of a linear operator, Acta Math. Sci. Ser. A, Chin. Ed., 34 (2014), 1922-1934.

5. A. Ammar, M. Zerai Dhahri, A. Jeribi, A characterization of S-essential spectrum by means of measure of non-strict-singularity and application, Azerb. J. Math., 5 (2015), no.1, 73-88.

6. C. Belabbaci, Jeribi essential spectrum, Lib. Math(N C). 37 (2018), 65-73.

7. C. Belabbaci, M. Aissani, M. Terbeche, S-essential spectra and measure of noncompactness, Math. Slovaca, 67 (2017), 1203-1212.

8. M. Benchohra, I. Medjadj, Measure of noncompactness and partial functional differential equations with state-dependent delay, Differ. Equ. Dyn. Syst., (2016), 1-17.

9. A. Jeribi, Spectral theory and application of linear operators and block operator matrices, Springer, 2015.

10. D. Edmunds, W.D. Evans, Spectral theory and differential operators, Oxford Math. Monogr., Clarendon Press, 1987.

11. M. Furi, M. Martelli, A. Vignoli, Contributions to the spectral theory for nonlinear operators in Banach spaces, Annali di Matematica Pura ed Applicata, 118 (1978), 229-294.

12. A. Jeribi, N. Moalla, S. Yengui, S-essential spectra and application to an example of transport operators, Math. Methods Appl. Sci., 37 (2014), 2341-2353.

13. R.D. Nussbaum, The radius of the essential spectrum, Duke Math. J., 37 (1970), 473-478.

14. E. Zeidler, Nonlinear functional analysis and its applications. I, Springer-Verlag, 1986.

Laboratory of Pure and Applied Mathematics

Department of mathematics, University of Laghouat, Algeria

c.belabbaci@lagh-univ.dz

Received 05.02.2020 